Submitted to Journal of Applied Physics

Preprint

\title{
ELECTRON MICROSCOPY OF SOME RARE EARTH-COBALT ALLOY MAGNETS
}

Raja K. Mishra and G. Thomas

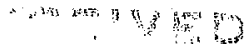
th shomenter

WAR B 1978

October 1977

LIBAARY AND DOCUMERTS SECTION

Prepared for the U. S. Department of Energy under Contract W-7405-ENG-48

\section{For Reference}

Not to be taken from this room

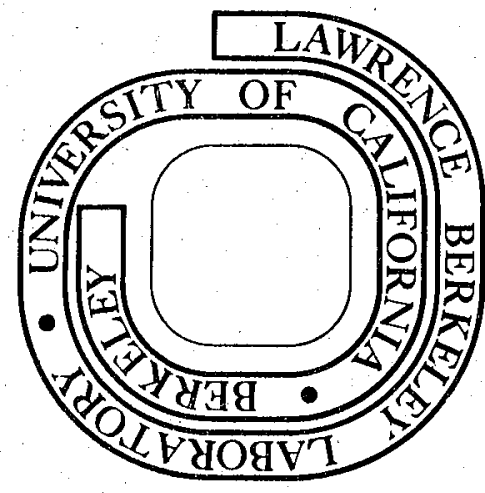


This report was prepared as an account of work sponsored by the United States Government. Neither the United States nor the Department of Energy, nor any of their employees, nor any of their contractors, subcontractors, or their employees, makes any warranty, express or implied, or assumes any legal liability or responsibility for the accuracy, completeness or usefulness of any information, apparatus, product or process disclosed, or represents that its use would not infringe privately owned rights. 
Raja K. Mishra and G. Thomas

Departhent of Materials Science and Mineral Engineering and Materials and Molecular Research Division, Lawrence Berkeley Laboratory, University of California, Berkeley, California 94720

\begin{abstract}
Transmission electron microscopy is being used to characterise the microstructural features in some 1:5 and $2: 17$ RE-Co magnets. In $2: 17$ compounds containing Fe and $\mathrm{Mn}_{\mathrm{n}}$, lamellae of hexagonal and other polytypic regions embedded in the rhombohedral matris are observed. It is shown that the interface between the hesagonal and rhombohedral phases can be described as a stacking fault with fault vector normal to the interfacial plane. APB's parallel to the prism planes in the rhombohedral structure have also been identified and characterised. In contrast, no planar faults have been observed in the $1: 5$ materials containing misch metal as the major rare-earth component. In these alloys extensive twinning and precipitation of a 2:17 Thombohedral phase are the major microstructural features observed. The influence of these microstructures on the magnetic properties are discussed.
\end{abstract}

\title{
INTRODUCTION
}

It is now accepted that, if the methods of processing, resulting microstructure, and corresponding properties can be correlated, a firm basis can be established for the design of materials with optimum properties. The primary role of microstructural characterisation in development work is the specification of those structures which result in the best process route-property relationships, and this implies also that detrimental microstructural (chemical) features have to be identified.

In recent years, a new class of magnets, based on raxe-earth cobalt (RE-Co) alloys with very high intrinsic coercivities ( $\mathrm{HC}_{\mathrm{f}}$ ) and maximum energy products [(BH) mars] have emerged. The study of their microstructures has received considerable attention $(1-5)$ in the last few years. Although much progress has been made in the understanding of microstructuremagnetic property relationships in some of the RE $-\mathrm{Co}$ magnets, most of the microstructural features (such as stacking faults, twins, second phases and interfaces etc.) have not been wel1-characterised, and their possible roles in the materials properties have yet to be established. In the present paper, an attempt 
has been made to discuss and identify a few of the destrable and undesirable microstructural features in some complex RE-Co alloys, with special emphasis on their characterisation. One of the alloys chosen for the present study is an economically produceable (1:5 RE:Co) magnet containing misch metal (nm) as the major rare earth component. Microstructural investigations of some 2:17 alloys containing Fe and Mn will also be discussed.

\section{EXPERTMENTAL}

Several sintered magnets were provided through the courtesy of Dr. D. Fry of General Motors Research Center, Warren, Michigan. One set of magnets had nominal powder composition m. $8 \mathrm{Sm} .2 \mathrm{Co} 5$ with misch metal (mm) composition $53 \%$ Ce, $25 \%$ La, $13 \% \mathrm{Nd}, 5 \% \mathrm{Pr}$ and $4 \%$ others. A second set of magnets had the composition $\mathrm{Sm}_{2}$ (Co.8 $\mathrm{Fe} . \mathrm{IM}^{\mathrm{Mn}} \mathrm{I}$ ) 17. These were prepared by sintering the magnetically aligned pellets for one hour at $1060^{\circ} \mathrm{C}$ and $1135^{\circ} \mathrm{C}$ respectively and were furnace cooled. The average grain size and densities were $\sim 2,5$ ums and $8.26,7.82 \mathrm{gms} / \mathrm{cc}$ respectively. The remanences and intrinsic coercivities determined by R.W. Lee of General Motors Research Lab. were $7.5 \mathrm{kG}$ and $14 \mathrm{kG}$ for the $1: 5$ alloys and $8.5 \mathrm{~kg}$ and $5.8 \mathrm{kG}$ for the 2:17 alloys respectively.

For microstructural characterisation, 3mm discs were spark cut from the sintered pellets. These were mechanically thinned and then ion milled until electron transparent. Thin folls were examined in a Hitachi HU-650 high voltage electron microscope operating at $650 \mathrm{kV}$. Some in situ hot stage observations were also made in a Philips EM-301 microscope.

\section{RESULTS ANO INTERPRETATTON}

a) Microstructure of $\mathrm{Sm}_{2}\left(\mathrm{Co} .8^{\mathrm{Fe}} \cdot 1^{\mathrm{Mn}} \mathrm{1}_{17} \mathrm{mag}-\right.$ nets. The grains in the sintered material were observed to have a $\mathrm{Th}_{2} \mathrm{Zn}_{17}$ type rhombohedral structure. As shown in Fig. 1 regions of hexagonal (Th2Ni17 structure type) material were quite often present. The boundary between these two phases can be described by a fault in the rhombohedral lattice with a fault vector normal to the boundary. By orienting the specinen so that the diffraction vector lies parallel to the edge-on boundary (i.e. such that $\vec{g} \cdot \vec{R}=0$ ), the contxast from the phase boundary can be made to disappear as shown in Fig. 1(b). This boundary has a Fault vector $=a / 3[0001]$, which corresponds to one basic building block (6) mismatch in the crystal structure. 
Another kind of stacking fault in the rhombohedral structure is shown in Fig. 2. The fringe contrast in Fig. 2(a) arises due to a phase change of $\pi$ in the electron beam as it crosses the fault. These faults are found to lie on the prism planes of the crystal and go out of contrast for diffracting conditions (7) such that $\vec{g} \cdot \vec{R}=0$ or an integer. The fringe contrast appears only when a systematic row with a higher order reflection ( $\geqslant \geqslant 4$ ) has been excited. Such contrast is consistent for faults having a $1 / 4<1010>$ displacement vector. Dynamical contrast calculations for the fringe profiles confirm this result. Such a fault can form due to impingement of regions nucleated in different stacking sequences and may be looked upon as an antiphase boundary (APB)。 Structurally, this planar fault disturbs the position of both Sm and "Co" atoms (unlike the faults in ferrimagnetic spinel ferrites where only cations are disturbed (7), which must affect the magnetic interactions locally。

Apart from these structural defects, the materials contain numerous inclusions as in Fig. 1. Furthermore, these inclusions axe dispersed non uniformly in only certain regions of the material.

Another common feature of the microstructure is shown in Fig. 3., in which a grain of the $2: 17$ phase contains periodic faulting. This striated microm structure (8) may be due to the presence of several polytypes (9) of the $2: 17$ phase as evidenced in the selected area diffraction patterns. Lattice imaging studies of these polytypes are currently in progress in order to establish the basic structures.

b) Microstructure of (mm) $.8 \mathrm{Sm} .2 \mathrm{Co}_{5}$ magnets: This material, unlike the $2: 17$ compounds, does not contain stacking faults of the type discussed earliex. Instead, these alloys contain a number of twinned grains. Part of such a grain is shown in Fig. 4. Twins are growth twins and the basal plane is the twin plane. It is believed that these occur upon recrys tallisation during sintering. On heating the specimen in the hot stage of a Philips EM-301 microscope at $860^{\circ} \mathrm{C}$, the twins are observed to grow in thickness in those grains.

As can be seen in Fig. $4(a)$ the resulting microstructure contains two different phases in the twinned and untwinned regions of the twinned grains. Careful analysis of the diffraction patterns from these regions shows that the microstructure consists of a 2:17 (rhombohedra1) phase which has precipitated from the 1:5 matrix. Upon continued annealing (in the hot stage of the EN-301), the second phase grows and a cellular microstructure (Fig. 4b) similar to that reported by Livingston and Martin (3), results. In 
grains where no twinning has occurred, small precipitates of hexagonal $2: 17$ phase form in the $1: 5$ matrix as shown in Fig. 5. These particles have a very slow growth rate and remain essentially unchanged in size during heating at $860^{\circ} \mathrm{C}$. In view of the $1: 5$ overall composition of these magnets, these observations can be explained if one assumes local composition differences between different grains.

\section{DISCUSSION}

The coercivity of the mm-containing $1: 5$ magnets is much higher than that of the $\mathrm{Mn}$, Fe doped $2: 17$ magnets. Our results of the microstructural inves tigation show that the $2: 17$ magnets contain predominantly the hexagonal $2: 17$ phase and other polytypic phases embedded in the rhombohedral matrix. These structures also contain planar defects such as APB's, stacking faults, interphase boundaries etc. which axe known to act as preferential sitts for reverse domain nucleation. Magnetisation data confirms that in these magnets, coercivity is in fact controlled by domain nucleation.

In the 1:5 magnets containing misch metal, the intrinsic coercivity is quite high. This is believed to be due to domain wall pinning by precipitates of the $2: 17$ phase (3) even when they are quite small in size. Also the occurrence of twins probably contributes to high coercivity (10). In order to investigate these proposals, further aging experiments are in progress so as to change the microstructure (dispersion of second phases) of the complex $1: 5$ magnets $\left(\mathrm{mm} .8^{\mathrm{Sm}} \cdot{ }_{2} \mathrm{Co}_{5}\right)$ : The peak aged properties have not been determined yet, but use of minstead of pure rare earth elements to prepare magnets with compar able properties is certainly attractive from an economical viewpoint.

The effect of alloying elements (such as Mn in this case) on the microstructures and magnetic properties of $\mathrm{RE}-\mathrm{Co}$ alloys is beginning to receive some attention $(2,11)$. In the present study, the role of Mn could not be ascertained precisely, although prolific faulting and polytypic transformations are observed. These feacures must be a consequence of the specific alloying additives used. Work is in progress to evaluate the role of some other alloying elements and establish the optimum anounts of alloying additions, as well as the accompanying heat treatments for best results. 


\section{ACKNOTLEDGENENTS}

This work was funded by ERDA through Lawrence Berkeley Laboratory. The matexials were kindly provided by D. Fry of the Physics Department, Rescarch Labs, General Motors Company.

\section{REFERENCES}

1. K. N. Melton and H. Nage1, "An Electron Microscope Study of Sm-Co-Cu-based Magnetic haterials with the $\mathrm{Sm}_{2} \mathrm{Co}_{17}$ Structure", J. Appl. Phys. 48, 2608-11, (1977).

2. K.N. Melton and R.S. Perkins, "Magnetic Properties of Sm: (Co, Cu) Alloys. I. Electron Microscopy," J. App 1. Phys. 47; 2671-2678, (1976).

3. J.D. Livingston and D.L. Martin, "Microstructure of Aged (Co,Cu,Fe) 7 Sm Magnets", J. Appl. Phys. $48,1350-54,(1977)$.

4. H. Nagel, A.J. Perry and A. Menth, "Hard-magnetic properties and Microstructure of $\mathrm{Sm}(\mathrm{Co}, \mathrm{Cu})_{z}$ Compounds", J.App 1. Phys. 47, 2662-70, (1976).

5. J.D. Livingston, "Domains in Sintered Co-Cu-Fe-Sm Magnets", J.Appl. Phys.46, 5259-5262.

6. C.W.Allen, K.C. Liao and A.E. Miller, "Fault Structures in Rare-Earth-Cobalt Intermetallics", Journal of the Less-Common Metals, 52, 109-115, (1977)。

7. R.K. Mishra and G. Thomas, "Lattice Defects in Lithium Ferrite Spine1", Proc. 6th Intn'1 Mat1's

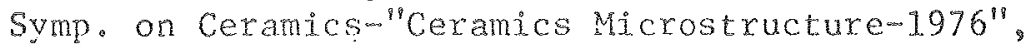
Ed. R.M. FuIrath and J.A. Pask, Westriew Press, Boulder, 71-81, (1977).

8. R.K. Mishra, F. Dizon and G。 Thomas, "Polycypism in Silicon Carbide", submitted to Phys. Stat. Sol., LBL Report 6932, 1-20, (1977).

9. C.W.Allen, D.L. Kuruzar and A.E. Miller, "Possible Magnetic Significance of Faults and Polytypes in $\mathrm{R}_{2} \mathrm{CO}_{17}$ Compounds", IEEE Trans. Magnetics, $M A Q-10,716-719,(1974)$.

10. H.R. Hilzinger, "The Influence of Planar Defects on Coercive Field of Haxd Magnetic Materials", App1. Phys. 12, 253-260, (1977).

11. T. Ojima, S. Tomizawa, T. Yoneyama and T. Hori, "Magnetic Properties of a New Type of Rare-Earth Cobalt Magnet: $\mathrm{Sm}_{2}(\mathrm{Co}, \mathrm{Cu}, \mathrm{Fe}, \mathrm{M}){ }_{17}$ ". TEEE Trans. Magnetics, MAG-13, 1317-19, (1977). 
Fig. 1. B.F. image of sintered (Co.gFe.19n.1) $17^{\mathrm{Sm}} 2$ showing regions of hexagonal 2:17 phase $(H)$ in rhombohedra1 2:17 matrix. The interfaces (A) correspond to faults in the rhombohedal structure and are out of contrast in $(b)(\vec{g} \cdot \vec{R}=0)$. Note the presence of inclusions $B$, scattered nonuniformly.

Fig. 2. a) B.F. image of $\pi$ faults in the rhombohedra1 2:17 phase. The associated fault vector $R=\frac{1}{4}\langle 0110>$. Faults are out of contrast in (b) where $\vec{g} \cdot \vec{R}=0$.

Fig. 3. Striated microstructure in 2:17 compounds. The corresponding $S A D$ pattern is shown in the insert.

Fig. 4. (a) Matxix dark field image of (Mn).85m.2 $\mathrm{Co}_{5}$ magnets showing twinning and $2: 17$ precipitate phase. (b) B.F. image of the microstructure of 1:5 magnets [as in (a)] upon annealing. The $2: 17$ precipitates grow and impinge giving rise to the cellular microstructure.

Fig. 5. B.F. image of isolated precipitates of a 2:17 phase in the $(\mathrm{Mn}) .8 \mathrm{Sm}_{2} \mathrm{CO}_{5}$ matrix. 
$00+0+942,610$

$-7-$
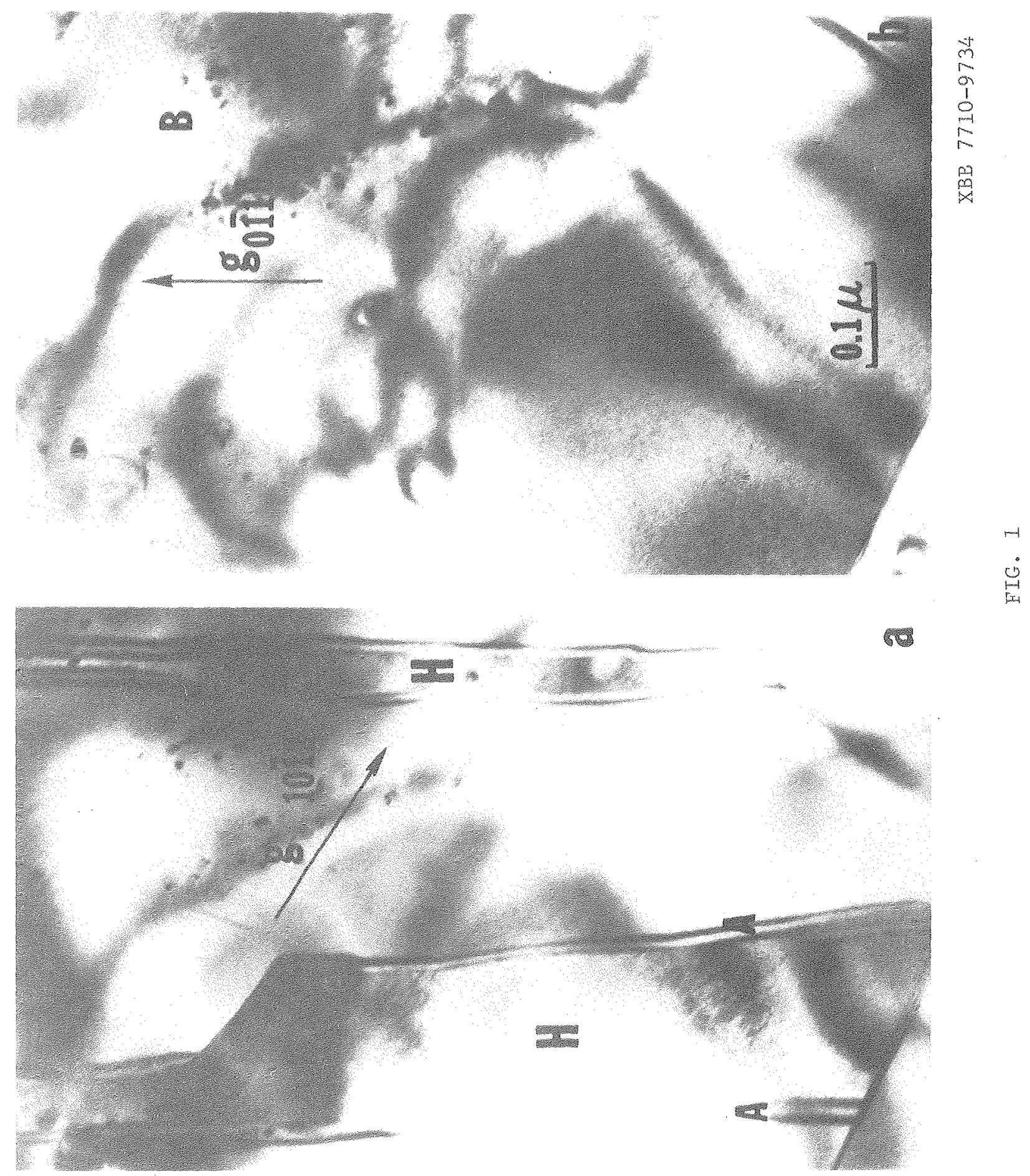

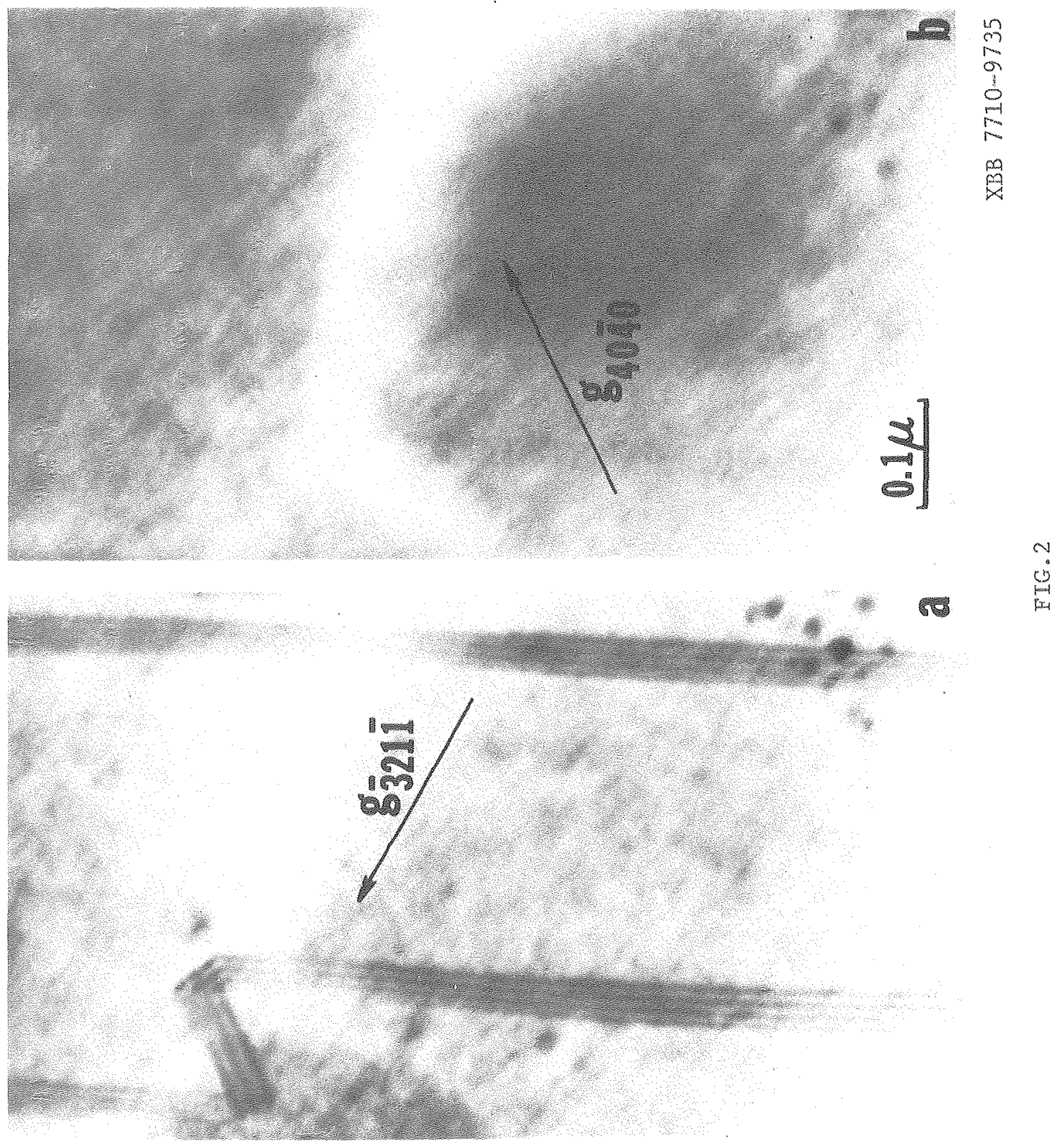
$04+4902041$

. 9

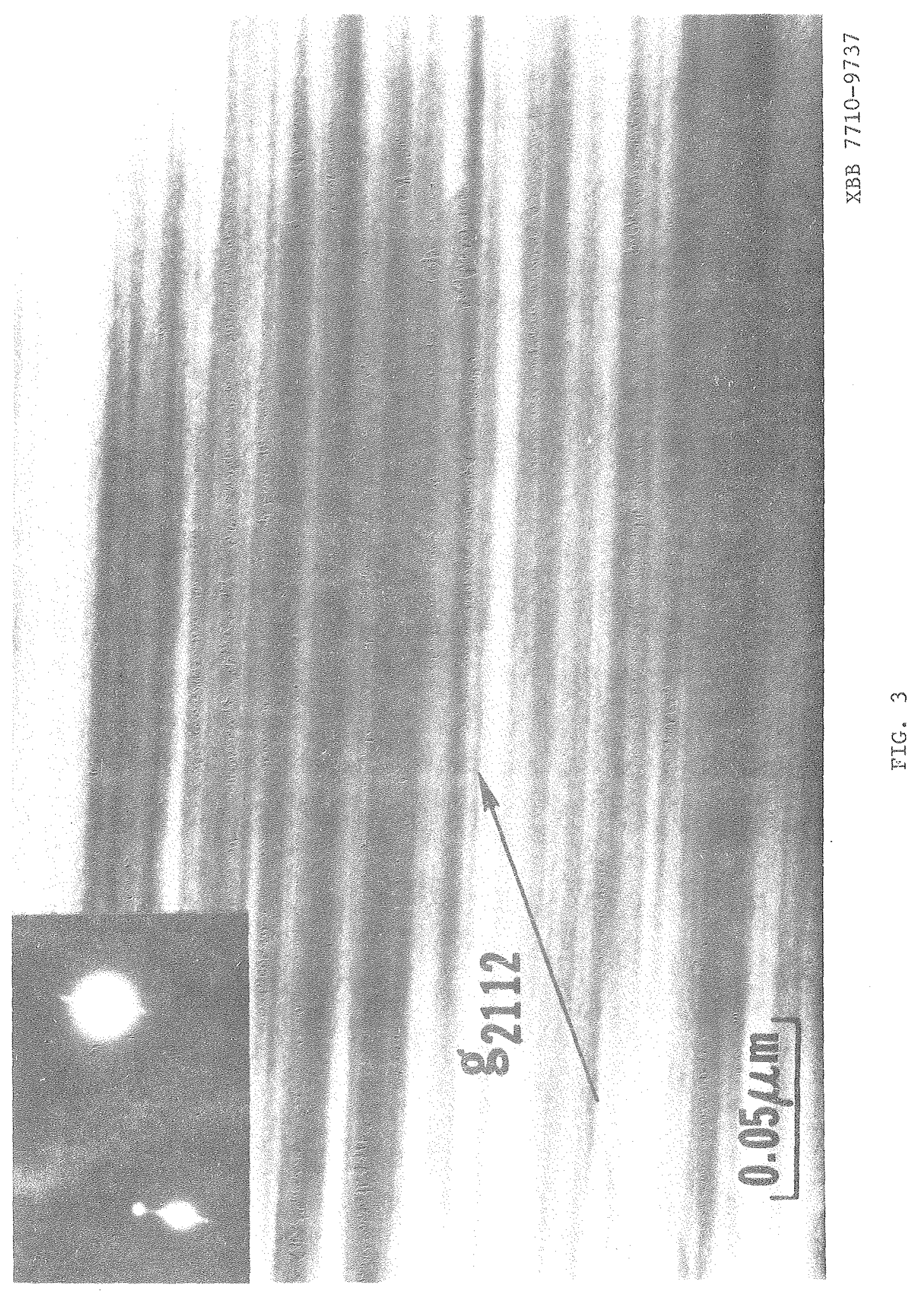




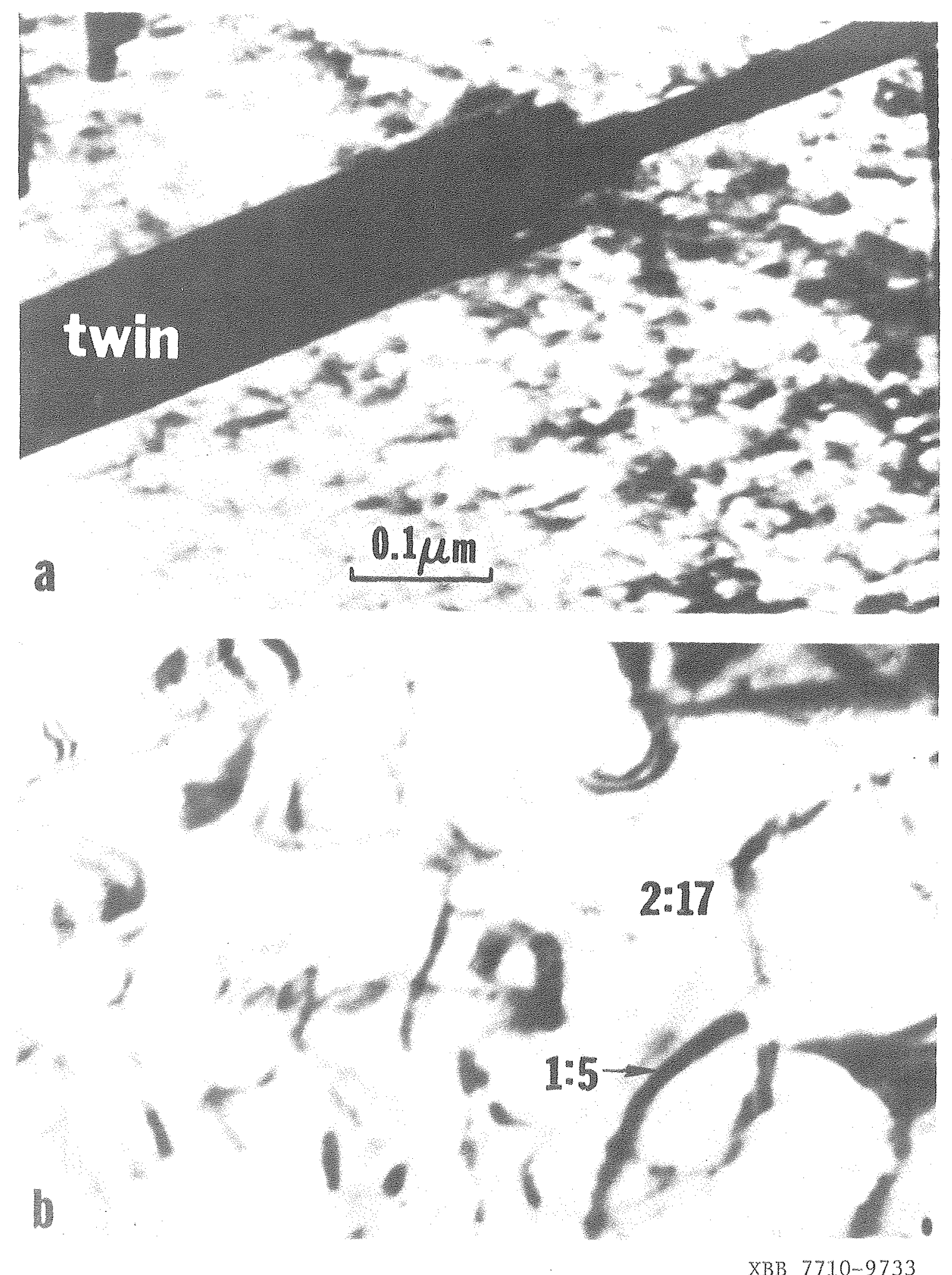

FIG. 4 


\section{$04+1 \% 902012$}

$-11-$
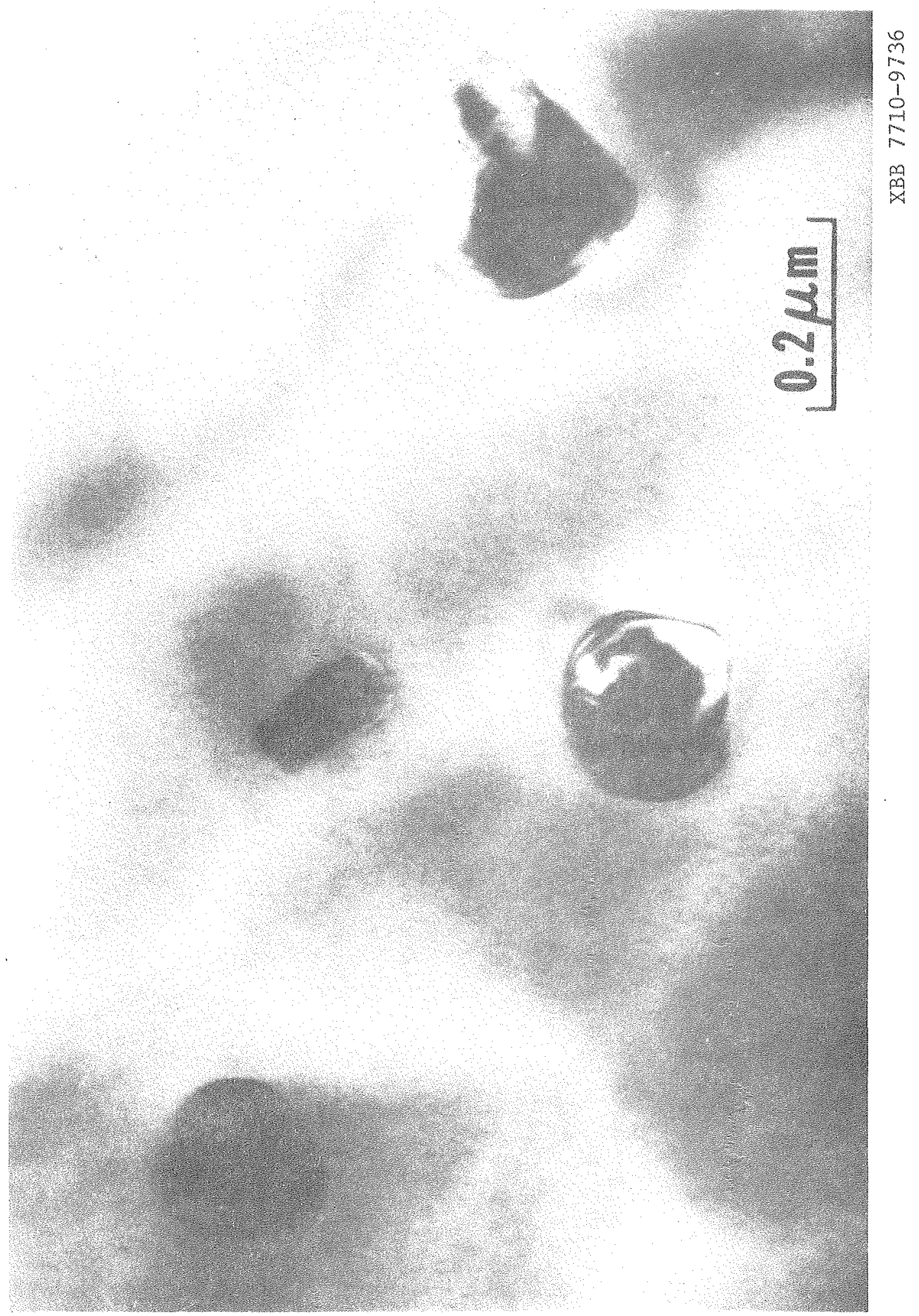

$\underbrace{n}_{n=1}$ 

This report was done with support from the United States Energy Research and Development Administration. Any conclusions or opinions expressed in this report represent solely those of the author(s) and not necessarily those of The Regents of the University of California, the Lawrence Berkeley Laboratory or the United States Energy Research and Development Administration. 